\title{
NOUVELLe
}

\section{Le pouce des mammifères, sous-produit de la colinéarité des gènes Hox}

\author{
Laboratoire de modélisation des systèmes biologiques, \\ UMR 7138 CNRS-Université Pierre et Marie Curie (Paris 6), \\ Université Pierre et Marie Curie, \\ 7, quai Saint Bernard, 75005 Paris, France. \\ legarrec@ccr.jussieu.fr
}

> La remarquable dextérité manuelle de l'homme est favorisée par l'existence d'un pouce en opposition avec les quatre autres doigts [1]. Cette indépendance du pouce est propre à certains primates, mais tous les mammifères ont hérité d'une morphologie ancestrale particulière: leur pouce est formé de deux phalanges, au lieu de trois pour les autres doigts. L'originalité du pouce de mammifère révèle ainsi une polarité de la main : celle-ci présente une extrémité antérieure (le pouce, doigt I), distincte de la partie postérieure (les doigts II à V). Cette asymétrie dérive d'une polarité encore plus ancienne du membre de vertébré [2]. Des travaux récents, réalisés chez la souris, ont montré que les gènes architectes Hox déterminent à la fois la polarité ancestrale du membre et celle des doigts de la main [3]. L'implication de ces gènes, étudiés par les biologistes du développement depuis trente ans, soulève une nouvelle question: l'arrangement des gènes Hox en complexes compacts sur le chromosome (Figure 1) a-t-il pu influencer la polarité de la main et l'apparition d'un doigt différent des autres, le pouce?

Un patron d'expression très particulier des gènes hoxd dans les doigts

Le laboratoire de Denis Duboule à Genève, en collaboration avec notre équipe de modélisateurs à Paris, vient de publier une étude qui apporte des éléments de réponse [4]. Ce même laboratoire avait précédemment montré que l'expression des gènes du complexe Hoxd dans les futurs doigts est régulée par deux éléments de contrôle, situés loin du complexe, côté centromérique (Figure 1) [5-7]. Le patron d'expression déterminé par cette régulation présente deux caractéristiques évidentes: (1) Alors que les gènes Hoxd9 à Hoxd12 sont exprimés dans les quatre doigts postérieurs, seul Hoxdl3 l'est dans les cinq doigts, y compris le pouce; (2) Le niveau d'expression décroît de façon colinéaire à l'ordre des gènes: Hoxdl3 est plus exprimé que Hoxd12, lequel est à son tour plus exprimé que Hoxdll, et ainsi de suite (Figure 1). Pour mieux comprendre ce mécanisme de régulation, les chercheurs ont étudié les effets de différents réarrangements du complexe Hoxd sur les niveaux d'expression des gènes du locus. Ces réarrangements, constitués de délétions ou duplications, sont réalisés par une technique de recombinaison méiotique [8]. La collaboration entre expérimentateurs et modélisateurs a suivi un processus itératif: les résultats de chaque nouvelle lignée de souris permettaient d'améliorer le modèle de régulation, alors que les prédictions du modèle aidaient au choix des réarrangements selon leur pouvoir discriminant.

\section{Identification du modèle}

de régulation des gènes du locus hoxd Les mesures d'ARN messagers transcrits par les sept gènes du locus, dans douze configurations différentes, ont conduit à un modèle dont les prédictions sont très proches des observations. Ce modèle décrit un mécanisme de régulation en deux étapes (Figure 2): (1) Dans un premier temps, les deux éléments de contrôle entrent en contact avec la région intergénique $\varepsilon v x 2-H o x d 13$, formant ainsi une double boucle d'ADN. Ce contact est d'autant plus fréquent que le nombre de gènes est important: en effet, les duplications de gènes ont pour conséquence une augmentation de

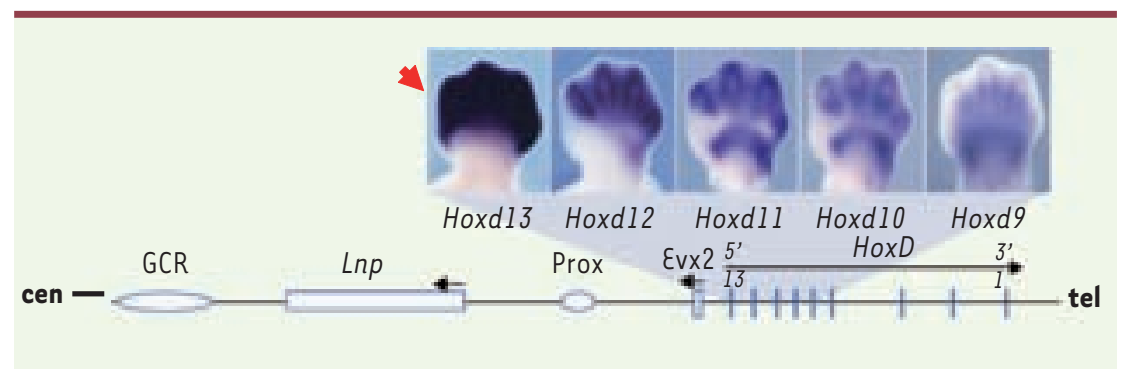

Figure 1. Le locus Hoxd et son expression colinéaire dans les doigts. En haut : les gènes Hoxd13 à Hoxd9 sont exprimés de façon colinéaire dans les futurs doigts d'un embryon de souris au stade 13,5 (jours post-coïtum) : $d 13$ est transcrit à un niveau élevé, alors que la transcription des gènes situés en 3' décroît progressivement (marquage par hybridation in situ). En bas : le locus Hoxd, situé sur le chromosome 2 de la souris, comprend dans le sens télomère (tel) vers centromère (cen), de droite à gauche : le complexe Hoxd proprement dit, le gène $\varepsilon v x 2$, l'élément de contrôle Prox, le gène Lnp et la région de contrôle GCR (global control region). La taille du locus est d'environ 300000 paires de bases. 
la quantité totale des ARN transcrits. (2) Dans un second temps, ces éléments activent la transcription de l'un des gènes, Lnp, Evx ou Hox, selon des probabilités reflétant les affinités respectives de leurs promoteurs. L'activation des gènes Hox est polarisée, les éléments de contrôle balayant le complexe à partir de Hoxdl3 vers Hoxd9, avec une probabilité de se fixer au passage sur l'un ou l'autre des promoteurs successifs.

La mise en évidence d'un tel mécanisme de régulation ouvre des perspectives tant en biologie cellulaire qu'en biologie de l'évolution. Alors qu'un nombre croissant d'éléments de contrôle distants sont identifiés chez les vertébrés [9], leur mode d'action demeure mal compris. Si le mécanisme proposé s'ap-

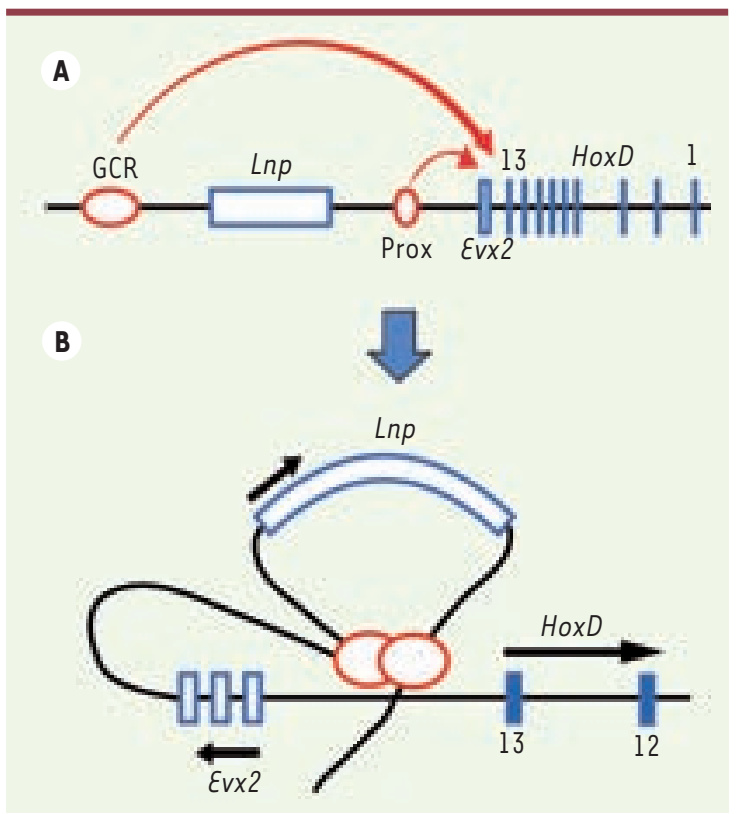

Figure 2. Un modèle de régulation du locus Hoxd en deux étapes.

A. Les éléments de contrôle GCR et Prox entrent en contact avec la région intergénique $\varepsilon v x 2-H o x d 13$, en formant deux boucles d'ADN. B. Ces éléments peuvent ensuite activer la transcription de Lnp, Evx2 ou des gènes Hox. L'activation des gènes Hox se fait de façon polarisée, les éléments de contrôle balayant le complexe dans le sens 5' vers 3'. plique à d'autres complexes de gènes, comme ceux des immunoglobulines ou des globines par exemple, la colinéarité d'expression pourrait être un phénomène courant.

\section{Et le pouce?}

L'évolution du pouce semble être une conséquence fortuite de la topologie et de la régulation du complexe Hoxd. Ces caractéristiques sont antérieures à l'apparition des mammifères : les quatre complexes Hox sont communs à l'ensemble des vertébrés; les deux éléments de contrôle existent, l'un chez les poissons téléostéens [5], I'autre chez les amphibiens et les oiseaux [7]. L'expression colinéaire des gènes Hoxd résulterait donc d'une topologie conservée au fil de l'évolution en raison de son importance pour d'autres fonctions, antérieures à l'apparition des doigts. Cette topologie est aussi caractérisée par l'existence de «déserts de gènes» de part et d'autre du complexe Hoxd, déserts qui comprennent un grand nombre de séquences non-codantes conservées chez les vertébrés. Le nombre de phalanges et la taille de chaque doigt dépendent de la dose totale de protéines Hox. Seul Hoxdl3 est exprimé dans la région du pouce, vraisemblablement parce que la topologie du locus favorise ce gène. Cette dose limitée de protéines Hox déterminerait la morphologie particulière du pouce, réduite à deux phalanges.
Quant aux gènes $L n p$ et $\varepsilon v x$, bien qu'ils soient exprimés dans les doigts en raison de leur position entre les éléments de contrôle et le complexe Hox, ils semblent n'y jouer aucun rôle.

La même publication présente également des mesures comparables effectuées dans le tubercule génital [4]. On sait aujourd'hui que le développement des doigts et des organes génitaux externes est régulé par les mêmes voies de signalisation. À l'exception de quelques délétions internes, le modèle dérivé des doigts permet aussi de prédire les niveaux d'expression dans le tubercule génital. Ce résultat semble confirmer l'existence d'un réseau ancestral de régulation partagé, réseau auquel se seraient ajoutés, au fil de l'évolution, des éléments particuliers à ces deux types d'organes. $\diamond$

The mammalian thumb, a by-product of Hox genes colinearity

\section{RÉFÉRENCES}

1. Napier J. Hands. Princeton: Princeton University Press, 1993.

2. Sordino P, Van der Hoeven F, Duboule D. Hox gene expression in teleost fins and the origin of vertebrate digits. Nature $1995 ; 375: 678-81$.

3. Tarchini B, Duboule D. Control of HoxD genes' colinearity during early limb development. Dev Cell $2006 ; 10: 1-11$.

4. Montavon T, Le Garrec JF, Kerszberg M, Duboule D. Modeling Hox gene regulation in digits: reverse collinearity and the molecular origin of thumbness. Genes Dev 2008 ; 22 : 346-59.

5. Spitz F, Gonzalez F, Duboule D. A global control region defines a chromosomal regulatory landscape containing the HoxD cluster. Cell 2003; 113 : 405-17.

6. Spitz F, Herkenne C, Hinard C, Duboule D. Un STRING lève le voile sur les mécanismes contrôlant l'expression des gènes Hox. Med Sci (Paris) 2006; 22: 14-6.

7. Gonzalez F, Duboule D, Spitz F. Transgenic analysis of Hoxd gene regulation during digit development. Dev Biol 2007 ; 306 : 847-59.

8. Hérault Y, Rassoulzadegan M, Cuzin F, Duboule D. Engineering chromosomes in mice through targeted meiotic recombination (TAMERE). Nat Genet 1998 ; $20: 381-4$.

9. Li Q, Peterson K, Stamatoyannopoulos G. Locus control regions. Blood $2002 ; 100$ : 3077-86. 